Annals of Pure and Applied Mathematics

Vol. 15, No. 1, 2017, 13-24

ISSN: 2279-087X (P), 2279-0888(online)

Published on 11 December 2017

www.researchmathsci.org

DOI: http://dx.doi.org/10.22457/apam.v15n1a2

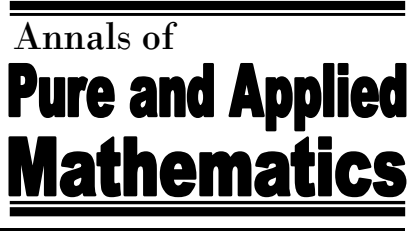

\title{
M/M/2/k Loss and Delay Interdependent Queueing Model with Controllable Arrival Rates, No Passing and Feedback
}

\author{
G. Rani $^{1}$ and V. Shanthi ${ }^{2}$ \\ ${ }^{1}$ PG \& Research Department of Mathematics, Seethalakshmi Ramaswami College \\ Tiruchirappalli - 620 002, Tamil Nadu, India. \\ E-mail: grsrc@yahoo.com \\ ${ }^{2}$ Department of Mathematics, Seethalakshmi Ramaswami College \\ Tiruchirappalli - 620 002, Tamil Nadu, India. \\ E-mail: varadarajanshanthi@ yahoo.com
}

Received 2 November 2017; accepted 5 December 2017

\begin{abstract}
In this paper M/M/2/k loss and delay queueing model with controllable arrival rates, 2 -server with identical service rates, no passing and feedback is considered. For this model, the steady state solution, the system characteristics are derived and the average waiting time for the two types of customers (Elective and Emergency) either with feedback or without feedback is obtained for varying arrival rates when the arrival and service processes are independent. The analytical results are numerically illustrated and the effect of the nodal parameters on the system characteristics are studied and relevant conclusion is presented.
\end{abstract}

Keywords: Controllable arrival rates, Finite capacity, Elective and Emergency Customers, No passing, Two server with parallel channels, Feedback.

AMS Mathematics Subject Classification (2010): 60K25, 68M20, 90B22

\section{Introduction}

Queuing system presents a concrete framework for design and analysis of practical applications. Queueing models provide the predictions of behaviour of systems such as waiting times, the average number of waiting customers and so forth. It is used in academic programs of Industrial Engineering, Computer Engineering etc., as well as in programs of Telecommunication and Computer Science. These predictions help us to anticipate situations to take appropriate measures to shorten the queues. Due to restriction of no passing the customers are allowed to depart from the system in the chronological order of their arrival either with feedback or without feedback. In the loss and delay queueing system the customers are classified into two classes. They are (a) Elective customers and (b) Emergency customers either with feedback or without feedback. The Elective customers have patience to form a queue and wait while the Emergency customers finding the server busy on their arrival, leave the system and are lost. But in many real life situations, the arrival and service patterns are interdependent.

Thiagarajan and Srinivasan [9] have analysed the M/M/1/k interdependent queueing model with controllable arrival rate. Rani and Srinivasan [5] have analysed 


\section{G. Rani and V. Shanthi}

$\mathrm{M} / \mathrm{M} / \mathrm{c} / \mathrm{k}$ interdependent queueing model with controllable arrival rates and feedback. Srinivasan and Thiagarajan [7] have discussed a finite capacity multiserver Poisson input queue with interdependent inter-arrival service time and controllable arrival rates. Rani and Srinivasan [6] have analysed $\mathrm{M} / \mathrm{M} / \mathrm{c} / \mathrm{k}$ loss and delay interdependent queueing model with controllable arrival rates, no passing and feedback. Kalyanaraman and Sumathy [3] have studied a feedback queue with multiple servers and batch service. Thangaraj and Shanthakumaran [8] studied a queue with a Markovian feedback. This paper is organized as follows: In section 2 a mathematical model for a M/M/2/k loss and delay with 2 -server in the same service rate interdependent queueing model with controllable arrival rates, no passing and feedback is described. In section 3 postulates of the model are stated. In section 4 the steady state equations of the model are framed. In section 5 the system characteristics are considered and the average waiting times for the two types of customers (Elective and Emergency) either with feedback or without feedback are obtained for varying arrival rates. And finally in the section 6 the analytical results are numerically illustrated, and the effect of the nodal parameters on the system characteristics are studied and relevant conclusion is presented.

\section{The diagrammatic representation of $\mathrm{M} / \mathrm{M} / 2 / \mathrm{k}$ loss and delay queueing system with} Bernoulli feedback

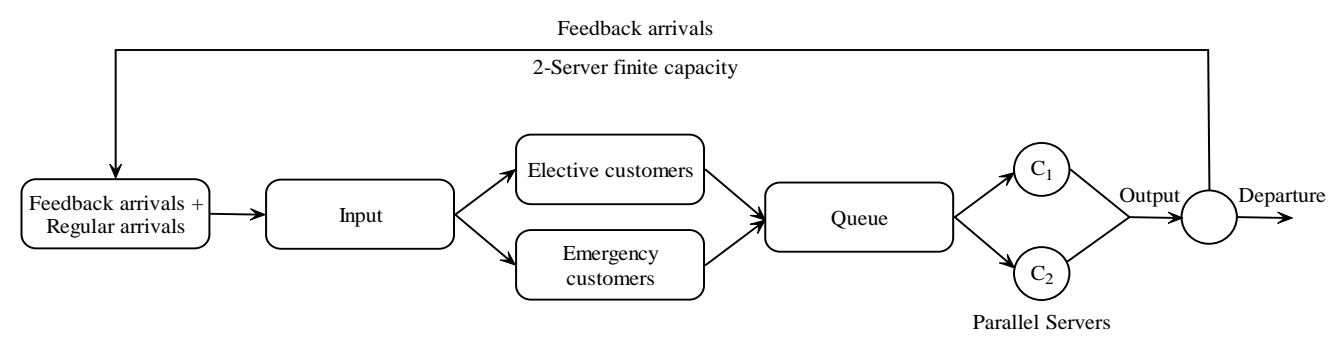

Figure 1:

- Due to restriction of no passing

- The elective customers have patience to form a queue and wait while the emergency customers finding the server busy on their arrival either with feedback or without feedback, leave the system and are lost.

\section{Description of the model}

Consider 2-server finite capacity loss and delay queueing system with controllable arrival rates, no passing and feedback. There are two types of customers (Elective and Emergency) arrive at the service station one by one according to a bivariate Poisson stream with arrival rates $\left(\lambda_{01}-\varepsilon\right),\left(\lambda_{02}-\varepsilon\right),\left(\lambda_{11}-\varepsilon\right),\left(\lambda_{12}-\varepsilon\right)(>0)$. There is 2-server providing service to all the arriving customers (Elective and Emergency) either with feedback or without feedback. Service times are independent and identically distributed exponential random variables with service rate $(\mu-\varepsilon)_{n}$. After the completion of each service, the Elective and Emergency customers can either join at the end of the queue with probability $\mathrm{p}$ or they can leave the system with probability $q, p+q=1$, the customer both newly arrived and those opted for feedback are served in the order in which they join the tail of the original queue. It is assumed that there is no difference between regular 
$\mathrm{M} / \mathrm{M} / 2 / \mathrm{k}$ Loss and Delay Interdependent Queueing Model with Controllable Arrival Rates,

No Passing and Feedback

arrivals and feedback arrivals. The Elective and Emergency customers are served according to the first come first served rule with following assumptions.

The arrival process $\left\{X_{1}(t)\right\}$ and the service process $\left\{X_{2}(t)\right\}$ of the system are correlated and follow a bivariate Poisson process given by

$$
\begin{aligned}
& P\left\{X_{1}(\mathrm{t})=x_{1}, X_{2}(\mathrm{t})=x_{2}\right\}=\frac{e^{-\left(\lambda_{i j}+\mu_{n}-\varepsilon\right)} \sum_{k=0}^{\min \left(x_{1}, x_{2}\right)}(\varepsilon t)^{k}\left[\lambda_{i j}-\varepsilon\right]^{x_{1}-k}\left[\mu_{n}-\varepsilon\right]^{x_{2}-k}}{k !\left(x_{1}-k\right) !\left(x_{2}-k\right) !} \\
& x_{1}, x_{2}=0,1,2,3, \ldots, \lambda_{i j}, \mu_{n}>0, i=0,1 \text { and } j=1,2 \\
& n=0,1,2, \ldots, c-1, c, c+1, \ldots, r-1, r, r+1, \ldots, R-1, R, R+1, \ldots, k-1, k
\end{aligned}
$$

with parameters $\lambda_{01}, \lambda_{02}, \lambda_{11}, \lambda_{12}, \mu_{n}$ and $\varepsilon$ as mean arrival rate of Elective customers, mean arrival rate of Emergency customers, when the system is in the faster rate of arrivals either with feedback or without feedback, mean arrival rate of Elective customers, mean arrival rate of Emergency customers, when the system is in the slower rate of arrivals either with feedback or without feedback, mean service rate and mean dependence rate (co-variance between arrival and service processes) respectively. defined as

Also the mean arrival rate and mean service rate when the system is of size $\mathrm{n}$ is

$$
\begin{aligned}
& \lambda_{i j}= \begin{cases}\lambda_{0 j} \delta ; & 0 \leq n<c ; j=1,2 \\
\lambda_{01} \delta ; & c \leq n \leq R-1 \\
\lambda_{1 j} \delta ; & r+1 \leq n \leq k ; j=1,2\end{cases} \\
& \mu_{n}= \begin{cases}n q \mu ; 0 \leq n<c \\
c q \mu ; c \leq n \leq k\end{cases}
\end{aligned}
$$

\section{The postulates of the model}

1. Probability that there is no arrival (Elective and Emergency) and no service completion during a small interval of time $\mathrm{h}$, when the system is with $\lambda_{i j}, i=0,1 \&$ $j=1,2$ faster (slower) rate of arrivals either with feedback or without feedback, is

$$
1-\left[\left(\lambda_{i j}-\varepsilon\right) \delta+p(\mu-\varepsilon)_{2}+q(\mu-\varepsilon)_{2}\right] h+o(h)
$$

2. Probability that there is one arrival (Elective and Emergency) and no service completion during a small interval of time $\mathrm{h}$, when the system is with $\lambda_{i j}, i=0,1 \&$ $j=1,2$ faster (slower) rate of arrivals either with feedback or without feedback, is

$$
\left(\lambda_{i j}-\varepsilon\right) \delta+o(h)
$$

3. Probability that there is no arrival (Elective and Emergency) and one service completion during a small interval of time $\mathrm{h}$, when the system is in faster or in slower rate of arrivals either with feedback or without feedback, is

$$
\left[q(\mu-\varepsilon)_{2}+p(\mu-\varepsilon)_{2}\right] h+o(h)
$$

4. Probability that there is one arrival (Elective and Emergency) and one service completion during a small interval of time $h$, when the system is either in faster $\left(\lambda_{i j}, i=0,1 \& j=1,2\right)$ or in slower rate of arrivals either with feedback or without feedback, is

$$
\left[\left(\lambda_{i j}-\varepsilon\right) \delta+p(\mu-\varepsilon)_{2}+q(\mu-\varepsilon)_{2}\right] h+o(h)
$$




\section{G. Rani and V. Shanthi}

\section{Steady state equations}

We observe that only $P_{n}(0)$ exists when $n=0,1,2, \ldots, c-1, c, c+1, \ldots, r-1, r$; both $P_{n}(0)$ and $P_{n}(1)$ exists when $n=r+1, r+2, \ldots, R-2, R-1$; only $P_{n}(1)$ exists when $n=R, R+1, \ldots, k$. Further $P_{n}(0)=P_{n}(1)=0$ if $n>k$.

The steady state equations which are written through the matrix of densities are given by

$$
\begin{aligned}
& {\left[\left(\lambda_{0 j}-\varepsilon_{j}\right) \delta\right] P_{0}(0)=q(\mu-\varepsilon) P_{1}(0)} \\
& {\left[\left(\lambda_{0 j}-\varepsilon_{j}\right) \delta+q(\mu-\varepsilon)\right] P_{1}(0)=\left[\left(\lambda_{0 j}-\varepsilon_{j}\right) \delta P_{0}(0)+2 q(\mu-\varepsilon) P_{2}(0)\right]} \\
& {\left[\left(\lambda_{0 j}-\varepsilon_{j}\right) \delta+2 q(\mu-\varepsilon)\right] P_{n}(0)=\left[\left(\lambda_{0 j}-\varepsilon_{j}\right) \delta P_{n-1}(0)+2 q(\mu-\varepsilon) P_{n+1}(0)\right]} \\
& n=2,3, \ldots, r-1 \\
& {\left[\left(\lambda_{0 \mathrm{j}}-\varepsilon_{j}\right) \delta+2 q(\mu-\varepsilon)\right] P_{r}(0)=\left(\lambda_{0 j}-\varepsilon_{j}\right) \delta P_{r-1}(0)+2 q(\mu-\varepsilon) P_{r+1}(0)} \\
& +2 q(\mu-\varepsilon) P_{r+1}(1)+2 p(\mu-\varepsilon) P_{r}(1) \\
& {\left[\left(\lambda_{0 j}-\varepsilon_{j}\right) \delta=+2 q(\mu-\varepsilon)\right] P_{n}(0)=\left(\lambda_{0 j}-\varepsilon_{j}\right) \delta P_{n-1}(0)+2 q(\mu-\varepsilon) P_{n+1}(0)} \\
& n=r+1, r+2, \ldots, R-2 \\
& {\left[\left(\lambda_{0 j}-\varepsilon_{j}\right) \delta+2 q(\mu-\varepsilon)\right] P_{R-1}(0)=\left(\lambda_{0 j}-\varepsilon_{j}\right) \delta P_{R-2}(0)} \\
& {\left[\left(\lambda_{l j}-\varepsilon_{j}\right) \delta+2 q(\mu-\varepsilon)\right] P_{r+1}(1)=2 q(\mu-\varepsilon) P_{r+2}(1)} \\
& {\left[\left(\lambda_{l j}-\varepsilon_{j}\right) \delta+2 q(\mu-\varepsilon)\right] P_{n}(1)=\left(\lambda_{l j}-\varepsilon_{j}\right) \delta P_{n-1}(1)+2 q(\mu-\varepsilon) P_{n+1}(1)} \\
& n=r+2, r+3, \ldots, R-1 \\
& {\left[\left(\lambda_{l j}-\varepsilon_{j}\right) \delta+2 q(\mu-\varepsilon)\right] P_{R}(1)=\left(\lambda_{l j}-\varepsilon_{j}\right) \delta P_{R-1}(1)+\left(\lambda_{0 j}-\varepsilon_{j}\right) \delta P_{R-1}(0)} \\
& +2 q(\mu-\varepsilon) P_{R+1}(1) \\
& {\left[\left(\lambda_{l j}-\varepsilon_{j}\right) \delta+2 q(\mu-\varepsilon)\right] P_{n}(1)=\left(\lambda_{l j}-\varepsilon_{j}\right) \delta P_{n-1}(1)+2 q(\mu-\varepsilon) P_{n+1}(1) \text {, }} \\
& n=R+1, R+2, \ldots, k-1
\end{aligned}
$$

$$
\left(\lambda_{1 j}-\varepsilon_{j}\right) \delta P_{k-1}(1)=2 q(\mu-\varepsilon) P_{k}(1)
$$

Let $\frac{\rho_{j}(0)}{2}=\frac{\left(\lambda_{0 j}-\varepsilon_{j}\right) \delta}{2 q(\mu-\varepsilon)}$ and $\frac{\rho_{j}(1)}{2}=\frac{\left(\lambda_{1 j}-\varepsilon_{j}\right) \delta}{2 q(\mu-\varepsilon)}$

where $\varepsilon_{1}+\varepsilon_{2}=\varepsilon, \lambda_{01}+\lambda_{02}=\lambda_{0},\left(\lambda_{01}-\varepsilon_{1}\right)+\left(\lambda_{02}-\varepsilon_{2}\right)=\lambda_{0}-\varepsilon$

and $\quad \lambda_{11}+\lambda_{12}=\lambda_{1},\left(\lambda_{11}-\varepsilon_{1}\right)+\left(\lambda_{12}-\varepsilon_{2}\right)=\left(\lambda_{1}-\varepsilon\right)$

From (1), (2) and (3), it can be shown that,

$$
P_{n}(0)=\frac{1}{2^{n-1}}\left(\rho_{j}(0)\right)^{n} P_{0}(0) ; n=1,2, \ldots, r
$$

Using the result (12) in (4) and (5) we get

$$
P_{n}(0)=\left\{\begin{array}{cc}
\frac{1}{2^{n-1}}\left(\rho_{j}(0)\right)^{n} P_{0}(0)-\left[\frac{1-\left(\frac{\rho_{j}(0)}{2}\right)^{n-r}}{1-\frac{\rho_{j}(0)}{2}}\right] P_{r+1}(1) ; \text { if } \frac{\rho_{j}(0)}{2} \neq 1 \\
n=r+1, r+2, \ldots R-1 & \text { if } \frac{\rho_{j}(0)}{2}=1 \\
2 P_{0}(0)-(n-r) P_{r+1}(1) ; &
\end{array}\right.
$$


M/M/2/k Loss and Delay Interdependent Queueing Model with Controllable Arrival Rates, No Passing and Feedback

Using equation (13) in (6) we get

$$
P_{r+1}(1)= \begin{cases}\frac{2\left(1-\frac{\rho_{j}(0)}{2}\right)\left(\frac{\rho_{j}(0)}{2}\right)^{R+r}}{\left(\frac{\rho_{j}(0)}{2}\right)^{r}-\left(\frac{\rho_{j}(0)}{2}\right)^{R}} P_{0}(0) ; & \text { if } \frac{\rho_{j}(0)}{2} \neq 1, j=1,2 \\ \frac{2}{R-r} P_{0}(0) ; & \text { if } \frac{\rho_{j}(0)}{2}=1, j=1,2\end{cases}
$$

Using the results (13) and (14) in (7) and (8), we get

$$
P_{n}(1)=\left\{\begin{array}{lc}
{\left[\frac{1-\left(\frac{\rho_{j}(1)}{2}\right)^{n-r}}{1-\frac{\rho_{j}(1)}{2}}\right] P_{r+1}(1) ;} & j=1,2 \text { if } \frac{\rho_{j}(1)}{2} \neq 1, \frac{\rho_{j}(0)}{2} \neq 1 \\
n=r+1, r+2, \ldots, R \\
(n-r) \mathrm{P}_{\mathrm{r}+1}(1) ; & \text { if } \frac{\rho_{j}(1)}{2}=\frac{\rho_{j}(0)}{2}=1 ; j=1,2
\end{array}\right.
$$

where $\mathrm{P}_{\mathrm{r}+1}(1)$ is given by (14)

From (9), (10) and (11) we have recursively derived that,

$$
P_{n}(1)= \begin{cases}{\left[\frac{\left(\frac{\rho_{j}(1)}{2}\right)^{n-R}-\left(\frac{\rho_{j}(1)}{2}\right)^{n-r}}{1-\frac{\rho_{j}(1)}{2}}\right] P_{r+1}(1) ;} & \text { if } \frac{\rho_{j}(0)}{2} \neq 1, \frac{\rho_{j}(1)}{2} \neq 1 ; j=1,2 \\ n=R+1, R+2, \ldots, k\end{cases}
$$

where $P_{r+1}(1)$ is given by (14).

\section{System characteristics}

In this section the following system characteristics are considered and their analytical results are derived.

1. The probability $P(0)$ that the system is in faster rate of [Elective and Emergency] arrivals either with feedback or without feedback.

2. The probability $P(1)$ that the system is in slower rate of [Elective and Emergency] arrivals either with feedback or without feedback.

3. The probability $P_{0}(0)$ that the system is empty.

4. The expected waiting time of the [Elective and Emergency] customers when the system is in the faster rate of arrivals either with feedback or without feedback.

5. The expected waiting time of the [Elective and Emergency] customers when the system is in slower rate of arrivals either with feedback or without feedback.

6. The difference between the expected waiting time of Elective and Emergency customers when the system is in the faster rate of arrivals either with feedback or without feedback. 


\section{G. Rani and V. Shanthi}

7. The difference between the expected waiting time of Elective and Emergency customers when the system is in slower rate of arrivals either with feedback or without feedback.

The probability that the system is in faster rate of arrivals [Elective and Emergency] either with feedback or without feedback is

$$
P(0)=\sum_{n=1}^{r} P_{n}(0)+\sum_{n=r+1}^{R-1} P_{n}(0)
$$

Using (12), (13) and (14) in (17), we get

$$
P(0)= \begin{cases}2\left[\frac{\frac{\rho_{j}(0)}{2}}{1-\frac{\rho_{j}(0)}{2}}-\frac{(R-r)\left(\frac{\rho_{j}(0)}{2}\right)^{R+r}}{\left(\frac{\rho_{j}(0)}{2}\right)^{r}-\left(\frac{\rho_{j}(0)}{2}\right)^{R}}\right] P_{0}(0) ; & \text { if } \frac{\rho_{j}(0)}{2} \neq 1 ; j=1,2 \\ 2[R+r] P_{0}(0) ; & \text { if } \frac{\rho_{j}(0)}{2}=1 ; j=1,2\end{cases}
$$

The probability that the system is in slower rate of arrivals (Elective and Emergency) either with feedback or without feedback is

$$
P(1)=\sum_{n=r+1}^{R} P_{n}(1)+\sum_{n=R+1}^{k} P_{n}(1)
$$

From (14), (15), (16) and (19), we get

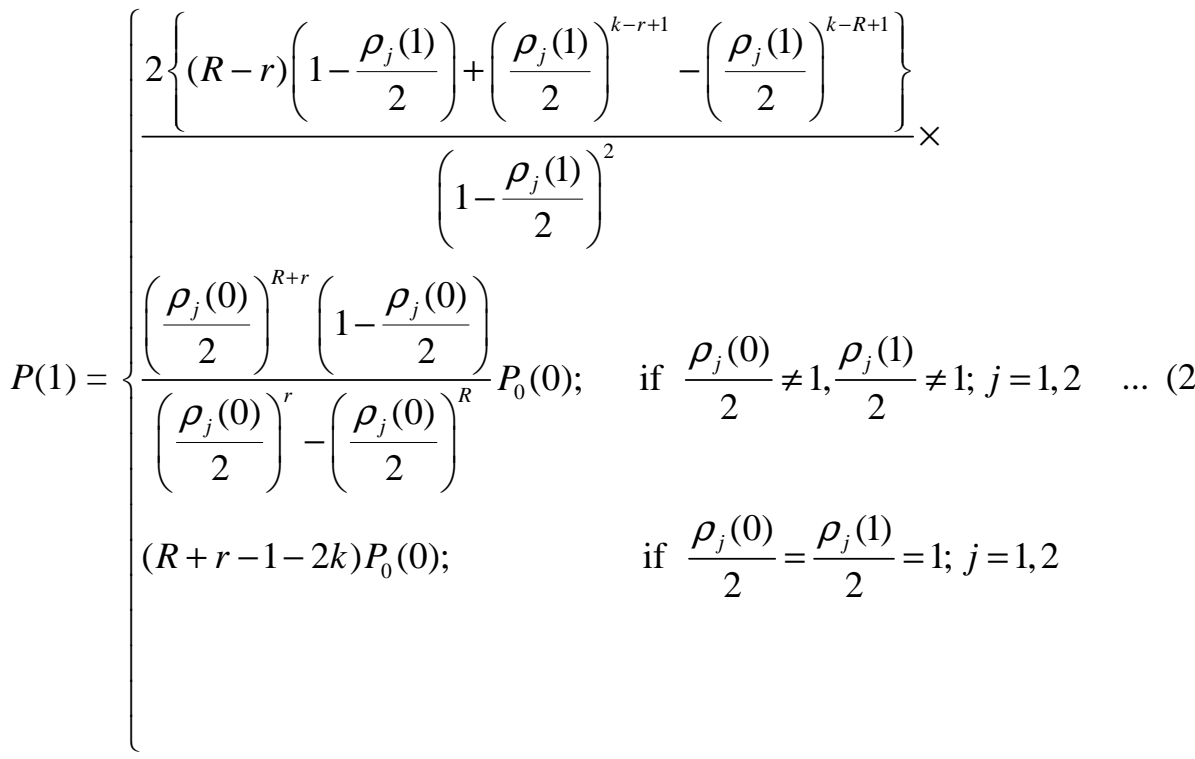

The probability $\left[P_{0}(0)\right]$ that the system is empty can be calculated from the normalizing condition

$$
P(0)+P(1)=1
$$


$\mathrm{M} / \mathrm{M} / 2 / \mathrm{k}$ Loss and Delay Interdependent Queueing Model with Controllable Arrival Rates, No Passing and Feedback

Using the results (18), (20) and (21), we get,

$$
P_{0}(0)=\left[2\left[\frac{\frac{\rho_{j}(0)}{2}}{1-\frac{\rho_{j}(0)}{2}}+\left(A+\frac{R-r}{1-\frac{\rho_{j}(1)}{2}}\right) B_{j}\right]\right]^{-1} ; j=1,2
$$

where $A=\frac{\left(\frac{\rho_{j}(1)}{2}\right)^{k-r+1}-\left(\frac{\rho_{j}(1)}{2}\right)^{k-R+1}}{\left(1-\frac{\rho_{j}(1)}{2}\right)^{2}}-\frac{(R-r)}{1-\frac{\rho_{j}(0)}{2}} ; j=1,2$

$$
B_{j}=\frac{\left(\frac{\rho_{j}(0)}{2}\right)^{R+r}\left(1-\frac{\rho_{j}(0)}{2}\right)}{\left(\frac{\rho_{j}(0)}{2}\right)^{r}-\left(\frac{\rho_{j}(0)}{2}\right)^{R}} ; j=1,2 .
$$

The expected waiting time of the Elective customers when the system is in the faster rate of arrivals either with feedback or without feedback is given by

$$
\mathrm{E}\left(\mathrm{W}_{\text {Ele } 0}\right)=\frac{1}{q(\mu-\varepsilon)}\left[\sum_{n=0}^{1} a_{n} P_{n}(0)+\left\{\sum_{n=2}^{r} P_{n}(0)+\sum_{n=r+1}^{R-1} P_{n}(0)\right\}\left(\frac{n-2+1}{2}+a_{1}\right)\right]
$$

where $a_{\mathrm{n}}= \begin{cases}0 ; & n=0 \\ \sum_{j=1}^{n} \frac{1}{j} ; & n=1,2,3, \ldots, k\end{cases}$

Using the results (12), (13), (14) and (23), we get,

$$
\begin{aligned}
\mathrm{E}\left(\mathrm{W}_{\mathrm{Ele} 0}\right) & =\frac{1}{q(\mu-\varepsilon)}\left[\left\{C_{1}+D_{1}\right\} P_{0}(0)+\left\{E_{1}+\frac{F_{1}}{2\left(1-\frac{\rho_{1}(0)}{2}\right)}+\frac{G_{1}}{2\left(1-\frac{\rho_{1}(0)}{2}\right)^{3}}\right\} P_{r+1}(1)\right] \\
& =\frac{1}{q(\mu-\varepsilon)}\left[C_{1}+D_{1}+2\left\{E_{1}+\frac{F_{1}}{2\left(1-\frac{\rho_{1}(0)}{2}\right)}+\frac{G_{1}}{2\left(1-\frac{\rho_{1}(0)}{2}\right)^{3}}\right\} B_{1}\right] P_{0}(0)
\end{aligned}
$$

where $C_{1}=\rho_{1}(0)+2\left(\frac{\rho_{1}(0)}{2}\right)^{2}\left[\frac{1-\left(\frac{\rho_{1}(0)}{2}\right)^{R-2}}{1-\frac{\rho_{1}(0)}{2}}\right]$

The expected waiting time of the Emergency customers when the system is in the faster rate of arrivals either with feedback or without feedback is given by 
G. Rani and V. Shanthi

$\mathrm{E}\left(\mathrm{W}_{\mathrm{Eme} 0}\right)=\frac{1}{q(\mu-\varepsilon)}\left[\sum_{n=0}^{1} a_{n+1} P_{n}(0)+\left\{\sum_{n=2}^{r} P_{n}(0)+\sum_{n=r+1}^{R-1} P_{n}(0)\right\}\left(\frac{n-2+1}{2}+a_{2}\right)\right]$

where $a_{n}= \begin{cases}0 ; & n=0 \\ \sum_{j=1}^{n} \frac{1}{j} ; & n=1,2,3, \ldots, k\end{cases}$

Using the results (12, (13), (14) and (25), we get

$$
\begin{aligned}
& \mathrm{E}\left(\mathrm{W}_{\mathrm{Eme} 0}\right)=\frac{1}{q(\mu-\varepsilon)}\left[C_{2}+D_{2}+2\left\{\frac{3}{2} E_{2}+\frac{F_{2}}{2\left(1-\frac{\rho_{2}(0)}{2}\right)}+\frac{G_{2}}{2\left(1-\frac{\rho_{2}(0)}{c}\right)^{3}}\right\} B_{2}\right] P_{0}(0) \\
& \text { where } C_{2}=\left[1+\frac{3}{2} \rho_{2}(0)\right]+3\left(\frac{\rho_{2}(0)}{2}\right)^{2}\left[\frac{1-\left(\frac{\rho_{2}(0)}{2}\right)^{R-2}}{1-\frac{\rho_{2}(0)}{2}}\right] \\
& D_{j}=\left[\frac{1-2\left(\frac{\rho_{j}(0)}{2}\right)^{R-1}+\left(\frac{\rho_{j}(0)}{2}\right)^{R-2}}{\left(1-\frac{\rho_{j}(0)}{2}\right)^{2}}-\frac{R\left(\frac{\rho_{j}(0)}{2}\right)^{R-2}}{\left(1-\frac{\rho_{j}(0)}{2}\right)}\right]\left(\frac{\rho_{j}(0)}{2}\right)^{2} ; j=1,2 \\
& E_{j}=\left[\frac{\frac{\rho_{j}(0)}{2}\left(1-\left(\frac{\rho_{j}(0)}{2}\right)^{R-r-1}\right)}{\left(1-\frac{\rho_{j}(0)}{2}\right)^{2}}-\frac{(R-r-1)}{1-\frac{\rho_{j}(0)}{2}}\right] ; j=1,2 \\
& F_{j}=(r-2) \frac{\rho_{j}(0)}{2}\left[\frac{1-\left(\frac{\rho_{j}(0)}{2}\right)^{R-r-1}}{1-\frac{\rho_{j}(0)}{2}}\right]-(r-2)(R-r-1)+\frac{(R-r)(R-r-1)}{2} ; j=1,2 \\
& G_{j}=\left(1-\frac{\rho_{j}(0)}{2}\right)^{2}+(R-r+1)\left(\frac{\rho_{j}(0)}{2}\right)^{R-r}-(R-r)\left(\frac{\rho_{j}(0)}{2}\right)^{R-r+1}-1 ; j=1,2
\end{aligned}
$$

where $P_{0}(0)$ and $P_{r+1}(1)$ are given by (22) and (14) respectively.

The expected waiting time of the Elective customer when the system is in the slower rate of arrivals either with feedback or without feedback is given by

$$
\mathrm{E}\left(\mathrm{W}_{\text {Ele } 1}\right)=\frac{1}{q(\mu-\varepsilon)}\left[\left(\sum_{n=r+1}^{R-1} P_{n}(1)+\sum_{n=R}^{k} P_{n}(1)\right)\left(\frac{n-2+1}{2}+a_{1}\right)\right]
$$

From (14), (15), (16) and (27), we get, 
$\mathrm{M} / \mathrm{M} / 2 / \mathrm{k}$ Loss and Delay Interdependent Queueing Model with Controllable Arrival Rates, No Passing and Feedback

$$
\begin{aligned}
& \mathrm{E}\left(\mathrm{W}_{\mathrm{Elel}}\right)=\frac{1}{q(\mu-\varepsilon)}\left[\frac { 1 } { 2 ( 1 - \frac { \rho _ { 1 } ( 1 ) } { 2 } ) } \left\{(r-2)(R-r-1)+\frac{(R-r-1)(R-r)}{2}+\frac{A_{1}^{\prime}}{\left(1-\frac{\rho_{1}(1)}{2}\right)}\right.\right. \\
& \left.\left.+\frac{B_{1}^{\prime}}{\left(1-\frac{\rho_{1}(1)}{2}\right)^{2}}\right\}+\frac{C_{1}^{\prime}}{\left(1-\frac{\rho_{1}(1)}{2}\right)}\right] P_{r+1}(1)
\end{aligned}
$$

The expected waiting time of the Emergency customers when the system is in the slower rate of arrivals either with feedback or without feedback is given by

$$
\mathrm{E}\left(\mathrm{W}_{\mathrm{Eme1}}\right)=\frac{1}{q(\mu-\varepsilon)}\left[\left(\sum_{n=r+1}^{R-1} P_{n}(1)+\sum_{n=R}^{k} P_{n}(1)\right)\left(\frac{n-2+1}{2}+a_{2}\right)\right]
$$

From (14), (15), (16) and (29), we get,

$$
\begin{aligned}
\mathrm{E}\left(\mathrm{W}_{\mathrm{Emel}}\right)=\frac{1}{q(\mu-\varepsilon)}\left[\frac { 1 } { 2 ( 1 - \frac { \rho _ { 2 } ( 1 ) } { 2 } ) } \left\{(r-2)(R-r-1)+\frac{(R-r-1)(R-r)}{2}+\frac{A_{2}^{\prime}}{\left(1-\frac{\rho_{2}(1)}{2}\right)}\right.\right. \\
\left.\left.\quad+\frac{B_{2}^{\prime}}{\left(1-\frac{\rho_{2}(1)}{2}\right)^{2}}\right\}+\left(\sum_{j=1}^{2} \frac{1}{j}\right) \frac{C_{2}^{\prime}}{\left(1-\frac{\rho_{2}(1)}{2}\right)}\right] P_{r+1}(1)
\end{aligned}
$$

where $A_{j}^{\prime}=(R-2)\left[1-\left(\frac{\rho_{j}(1)}{2}\right)^{k-R+1}+\left(\frac{\rho_{j}(1)}{2}\right)^{k-r+1}\right]-(r-2) \frac{\rho_{j}(1)}{2} ; j=1,2$

$$
\begin{aligned}
B_{j}^{\prime}=1-\left(\frac{\rho_{j}(1)}{2}\right)^{R-r}+(k-R+2)\left[\left(\frac{\rho_{j}(1)}{2}\right)^{k-r+1}-\left(\frac{\rho_{j}(1)}{2}\right)^{k-R+1}\right] \\
+(k-R+1)\left[\left(\frac{\rho_{j}(1)}{2}\right)^{k-R+2}-\left(\frac{\rho_{j}(1)}{2}\right)^{k-r+2}\right] ; j=1,2 \\
C_{j}^{\prime}=(R-r-1)+\left[1-\frac{\rho_{j}(1)}{2}-\left(\frac{\rho_{j}(1)}{2}\right)^{k-R+1}+\left(\frac{\rho_{j}(1)}{2}\right)^{k-r+1}\right] ; j=1,2
\end{aligned}
$$

where $P_{0}(0)$ and $P_{r+1}(1)$ are given by (22) and (14) respectively.

The difference between the expected waiting time of Elective customers and Emergency customers when the system is in the faster rate of arrivals either with feedback or without feedback is given by

$$
\Delta_{0}=\mathrm{E}\left(\mathrm{W}_{\text {Eme0 }}\right)-\mathrm{E}\left(\mathrm{W}_{\text {Ele } 0}\right)
$$


G. Rani and V. Shanthi

Using the results (24) and (26) in (31) we get

$$
\begin{aligned}
\Delta_{0}=\frac{1}{q(\mu-\varepsilon)}\left[\left(C_{2}-C_{1}\right)\right. & +\left(D_{2}-D_{1}\right)+2\left\{\left[\left(\sum_{j=1}^{2} \frac{1}{j}\right) E_{2}-E_{1}\right]+\frac{1}{2}\left[\frac{F_{2}}{\left.1-\frac{\rho_{2}(0)}{2}-\frac{F_{1}}{1-\frac{\rho_{1}(0)}{2}}\right]}\right.\right. \\
& \left.\left.+\frac{1}{2}\left[\frac{G_{2}}{\left(1-\frac{\rho_{2}(0)}{2}\right)^{3}}-\frac{G_{1}}{\left(1-\frac{\rho_{1}(0)}{2}\right)^{3}}\right]\right\}\left(B_{2}-B_{1}\right)\right] P_{0}(0)
\end{aligned}
$$

The difference between the expected waiting time of Elective customers and Emergency customers when the system is in the slower rate of arrivals either with feedback or without feedback is

$$
\begin{aligned}
& \Delta_{1}=\mathrm{E}\left(\mathrm{W}_{\text {Eme } 1}\right)-\mathrm{E}\left(\mathrm{W}_{\text {Ele } 1}\right) \\
& \Delta_{1}=\frac{1}{q(\mu-\varepsilon)}\left[\frac{(R+r-4)(R-r-1)}{4} \frac{\left[\frac{\rho_{2}(1)}{2}-\frac{\rho_{1}(1)}{2}\right]}{\left(1-\frac{\rho_{2}(1)}{2}\right)\left(1-\frac{\rho_{1}(1)}{2}\right)}\right. \\
& +\left[\frac{A_{2}^{\prime}}{\left(1-\frac{\rho_{2}(1)}{2}\right)}-\frac{A_{1}^{\prime}}{\left(1-\frac{\rho_{1}(1)}{2}\right)}\right]+\left[\frac{B_{2}^{\prime}}{\left(1-\frac{\rho_{2}(1)}{2}\right)^{2}}-\frac{B_{1}^{\prime}}{\left(1-\frac{\rho_{1}(1)}{2}\right)^{2}}\right] \\
& \left.+\left[\left(\sum_{j=1}^{2} \frac{1}{j}\right) \frac{C_{2}^{\prime}}{\left(1-\frac{\rho_{2}(1)}{2}\right)}-\frac{C_{1}^{\prime}}{\left(1-\frac{\rho_{1}(1)}{2}\right)}\right]\right] P_{r+1}(1)
\end{aligned}
$$

where $P_{0}(0)$ and $P_{r+1}(1)$ are given by (22) and (14) respectively.

\section{Numerical illustrations}

For various values of $\lambda_{01}, \lambda_{02}, \lambda_{11}, \varepsilon, \mu$ and fixed values of $c, R, r, k$, the values of $P_{0}(0), P(0)$ and $P(1)$ are computed and tabulated by taking $p=q=\frac{1}{2}, c=2, r=6, R=14, k=22$.

Table 1:

\begin{tabular}{|c|c|c|c|c|c|c|c|}
\hline$\lambda_{\boldsymbol{1} 1}$ & $\lambda_{\boldsymbol{0} 2}$ & $\boldsymbol{\lambda}_{\boldsymbol{1 1}}$ & $\boldsymbol{\varepsilon}$ & $\boldsymbol{\mu}$ & $\boldsymbol{P}_{\mathbf{0}}(\mathbf{0})$ & $\boldsymbol{P}(\mathbf{0})$ & $\boldsymbol{P}(\mathbf{1})$ \\
\hline 7 & 5 & 6 & 0.5 & 14 & 0.538482476 & 0.999727953 & $2.720456118 \times 10^{-04}$ \\
\hline 8 & 7 & 9 & 1.0 & 16 & 0.571168139 & 0.999331448 & $2.429658053 \times 10^{-04}$ \\
\hline 9 & 7 & 8 & 0.5 & 18 & 0.529430081 & 0.999688968 & $3.110315987 \times 10^{-04}$ \\
\hline 6 & 5 & 7 & 1.0 & 12 & 0.599981582 & 0.999814729 & $1.852708133 \times 10^{-04}$ \\
\hline
\end{tabular}


$\mathrm{M} / \mathrm{M} / 2 / \mathrm{k}$ Loss and Delay Interdependent Queueing Model with Controllable Arrival Rates, No Passing and Feedback

For various values of $\lambda_{01}, \lambda_{02}, \lambda_{12}, \varepsilon, \mu$ and fixed values of $c, R, r, k$, the values of $P_{0}(0)$, $P(0)$ and $P(1)$ are computed and tabulated by taking $p=q=\frac{1}{2}, c=2, r=6, R=14, k=22$.

Table 2:

\begin{tabular}{|c|c|c|c|c|c|c|c|}
\hline $\boldsymbol{\lambda}_{\mathbf{0 1}}$ & $\boldsymbol{\lambda}_{\mathbf{0 2}}$ & $\boldsymbol{\lambda}_{\mathbf{1 2}}$ & $\boldsymbol{\varepsilon}$ & $\boldsymbol{\mu}$ & $\boldsymbol{P}_{\mathbf{0}}(\mathbf{0})$ & $\boldsymbol{P}(\mathbf{0})$ & $\boldsymbol{P}(\mathbf{1})$ \\
\hline 7 & 5 & 4 & 0.5 & 14 & 1.000000337 & 0.999996989 & $3.011139509 \times 10^{-06}$ \\
\hline 8 & 7 & 6 & 1.0 & 16 & 0.750002418 & 0.999970990 & $2.900985846 \times 10^{-05}$ \\
\hline 9 & 7 & 6 & 0.5 & 18 & 0.846154757 & 0.999988191 & $1.18083851 \times 10^{-05}$ \\
\hline 6 & 5 & 4 & 1.0 & 12 & 0.875001084 & 0.999991337 & $8.661858418 \times 10^{-06}$ \\
\hline
\end{tabular}

For various values of traffic intensities $\rho_{1}(0), \rho_{1}(1), \rho(0)$ and fixed values of $c, R$, $r, k$, the values of $P_{0}(0), \mathrm{E}\left(\mathrm{W}_{\text {Ele } 0}\right)$ and $\mathrm{E}\left(\mathrm{W}_{\text {Ele } 1}\right)$ are computed and tabulated by taking $p=q=\frac{1}{2}, c=2, r=6, R=14, k=22$.

Table 3:

\begin{tabular}{|c|c|c|c|c|c|}
\hline $\boldsymbol{\rho}_{\mathbf{1}}(\mathbf{0})$ & $\boldsymbol{\rho}_{\mathbf{1}}(\mathbf{1})$ & $\boldsymbol{\rho}(\mathbf{0})$ & $\boldsymbol{P}_{\boldsymbol{0}}(\mathbf{0})$ & $\mathbf{E}\left(\mathbf{W}_{\text {Ele0 }}\right)$ & $\mathbf{E}\left(\mathbf{W}_{\text {Ele1 }}\right)$ \\
\hline 0.9629629 & 0.8148148 & 1.6296296 & 0.538482476 & 0.216822959 & $2.305440288 \times 10^{-04}$ \\
\hline 0.9333333 & 1.0666666 & 1.7333333 & 0.571168139 & 0.191642350 & $1.954836409 \times 10^{-04}$ \\
\hline 0.9714285 & 0.8571428 & 1.7142857 & 0.529430081 & 0.168165285 & $2.048172575 \times 10^{-04}$ \\
\hline 0.9090909 & 1.0909090 & 1.6363636 & 0.599981582 & 0.257493967 & $2.047189430 \times 10^{-04}$ \\
\hline
\end{tabular}

For various values of traffic intensities $\rho_{2}(0), \rho_{2}(1), \rho(0)$ and fixed values of $c, R$, $r, k$, the values of $P_{0}(0), \mathrm{E}\left(\mathrm{W}_{\mathrm{Eme0}}\right)$ and $\mathrm{E}\left(\mathrm{W}_{\mathrm{Emel}}\right)$ are computed and tabulated by taking $p=q=\frac{1}{2}, c=2, r=6, R=14, k=22$.

Table 4:

\begin{tabular}{|c|c|c|c|c|c|}
\hline $\boldsymbol{\rho}_{2}(\mathbf{0})$ & $\boldsymbol{\rho}_{2}(\mathbf{1})$ & $\boldsymbol{\rho}(\mathbf{0})$ & $\boldsymbol{P}_{\mathbf{0}}(\mathbf{0})$ & $\mathbf{E}\left(\mathbf{W}_{\text {Eme0 }}\right)$ & $\mathbf{E}\left(\mathbf{W}_{\text {Eme1 }}\right)$ \\
\hline 0.6666666 & 0.5185185 & 1.6296296 & 1.000000337 & 0.407405101 & $2.672235613 \times 10^{-06}$ \\
\hline 0.8000000 & 0.6666666 & 1.7333333 & 0.750002418 & 0.344406862 & $3.873679121 \times 10^{-05}$ \\
\hline 0.7428571 & 0.6285714 & 1.7142857 & 0.846154757 & 0.301889433 & $8.168855381 \times 10^{-06}$ \\
\hline 0.7272727 & 0.5454545 & 1.6363636 & 0.875001084 & 0.483755854 & $3.944311137 \times 10^{-06}$ \\
\hline
\end{tabular}

The numerical results for the expected waiting times of the two classes of customers (Elective and Emergency) for varying arrival rates (faster and slower) either with feedback or without feedback has been provided in the Tables 1,2, 3 and 4. 


\section{G. Rani and V. Shanthi}

From Tables 1 and 2 the following observations can be made:

i. In the long run, the probability that the system to be in the faster rate of arrivals either with feedback or without feedback is nearly unity. But the probability that the system to be in the slower rate of arrivals either with feedback or without feedback is very small.

ii. When the system is in the faster rate of arrivals either with feedback or without feedback, the probability that the system size is zero decrease when the arrival rate of the Elective customers or the arrival rate of the Emergency customers increases.

\section{From Tables 3 and 4 the following observations can be made:}

iii. Either the system is in the faster rate of arrivals or in the slower rate of arrivals either with feedback or without feedback, the expected waiting time of Elective customers $\left[\mathrm{E}\left(\mathrm{W}_{\mathrm{Ele} 0}\right)\right]$ and the expected waiting time of emergency customers $\left[\mathrm{E}\left(\mathrm{W}_{\mathrm{Eme} 1}\right)\right]$ increase as the parameters $\rho_{1}(0)$ and $\rho_{2}(0)$ increases.

iv. When the system is in the faster rate of arrivals either with feedback or without feedback, the expected waiting time of Elective customers $\left[\mathrm{E}\left(\mathrm{W}_{\mathrm{Ele} 0}\right)\right]$ and the expected waiting time of Emergency customers $\left[\mathrm{E}\left(\mathrm{W}_{\mathrm{Eme} 0}\right)\right]$ decrease as the traffic intensities $\rho_{1}(0)$ and $\rho_{2}(0)$ increases.

v. When the system is in the slower rate of arrivals either with feedback or without feedback, the expected waiting time of Elective customers $\left[\mathrm{E}\left(\mathrm{W}_{\text {Ele } 1}\right)\right]$ and the expected waiting time of Emergency customers $\left[\mathrm{E}\left(\mathrm{W}_{\mathrm{Eme} 1}\right)\right]$ increase as the traffic intensity $\rho_{1}(1)$ and $\rho_{2}(1)$ increases.

\section{REFERENCES}

1. D. Gross and C.M. Harris, Fundamentals of Queueing Theory, John Wiley, New York, 1974.

2. D. Gross and C.M. Harris, Fundamentals of Queueing Theory, Third Edition, Wiley Intersciences, New York, 1998.

3. R. Kalyanaraman and S. Sumathy, A Feedback Queue with multiservers and Batch Service, International Review of Pure and Applied Mathematics, 5(1) (2009) 23-27.

4. J. Medhi, Stochastic Models in Queueing Theory, 2/e Academic Press, An imprint of Elsevier, 2006.

5. G. Rani and A. Srinivasan, M/M/c/k Interdependent Queueing Model with controllable Arrival Rates and feedback, IJAMAA, 7(2) (2012) 131-141.

6. G. Rani and A. Srinivasan, M/M/c/k Loss and Delay Interdependent Queueing Model with controllable Arrival Rates, No passing and Feedback, International Journal of Mathematical Sciences and Engineering Applications, 7(IV) (2013) 403-419.

7. A.Srinivasan and M. Thiagarajan, A finite Capacity multi-server Poisson input Queue with Interdependent inter-arrival service time and controllable arrival rates, Bulletin of Calcutta Mathematical Society, 99(2) (2007) 173-182.

8. V. Thangaraj and A. Shanthakumaran, A Queue with Markovian feedback, Int. Jr. of Infor. and Manage, Sci., 6(4) (1995) 35-45.

9. M. Thiagarajan and A. Srinivasan, The M/M/1/k interdependent queueing model with controllable arrival rate, Int. Journal of Management and Systems, 22(1) (2006) 23-24. 